a local order-to-disorder transition or melting that allows the polymer to flow ${ }^{8}$ making it possible for the tip to make an impression in the polymer. The glass transition temperatures for both the ordered and the disordered block copolymers are well above room temperature, so the mark in the polymer made by the tip is frozen in place when the tip is removed and the pressure decreased. This mark can be used for information storage. Kim and co-workers produced features that were $2 \mathrm{~nm}$ deep with a pitch of $37 \mathrm{~nm}$, which translates into a storage density of $\sim 1 \mathrm{Tbit}^{-2}$. The elegance of this process is that entropy (pressure) rather than enthalpy (heat) enables the writing to be done at room temperature.

The maximum storage density achievable will depend on the sharpness of the tip and also on the amount of surface energy that is needed to generate such small features. Sharper tips lead to smaller features and higher storage densities, but nature abhors such small features and a Laplace pressure operates to smooth the surface. If the temperature is not sufficiently below the $T_{\mathrm{g}}$ of the polymer, the polymer will relax and the features will be lost. This is where a second important characteristic of block copolymers comes into effect. The different interactions of the blocks with the substrate and the difference in the surface energies of the blocks cause the blocks to segregate. However, as the blocks are linked together, in thin films this segregation leads to a multilayered structure consisting of alternating layers of the two blocks 9 .

The films used by Kim and co-workers initially consisted of alternating layers of polystyrene, with $T_{\mathrm{g}} \sim 100^{\circ} \mathrm{C}$, and PnPMA $\left(T_{\mathrm{g}} \sim 7^{\circ} \mathrm{C}\right)$. The $T_{\mathrm{g}}$ of the mixed block copolymer is $\sim 70^{\circ} \mathrm{C}$, so the marks made by the tip were frozen into place during operation at room temperature and atmospheric pressure. Outside the indentation region the polymer is ordered and the flow is retarded, thus removing the need for crosslinkers (which are needed when homopolymer films are used ${ }^{10}$ ). The multilayered structure of the film also means that the film thickness at any point is defined in terms of $L_{0}$ (see Fig. 1 and ref. 9). This means that the planarity of the film surface is guaranteed by this layering as long as the thickness of the original film placed on the substrate is commensurate with $L_{0}$. If not, a terraced surface topography will form with steps $L_{0}$ in height ${ }^{9}$.

The results of Kim and co-workers ${ }^{7}$ show clear advantages in using block copolymers that show lower disorder-to-order transition or closed-loop behaviour. With advantages also come disadvantages. Lower disorderto-order transitions and/or closed-loop behaviour are only seen in some of the polystyrene-block-poly(n-alkylmethacrylates). Although the syntheses of this family of block copolymers is well known, this limitation is disadvantageous, particularly as the $\operatorname{poly}(n$-alkylmethacrylates) have low values of $T_{\mathrm{g}}$ that can give rise to a long-term instability of the written features. Furthermore, methacrylate-based polymers can degrade. However, these potential drawbacks should not detract from the importance of the work of Kim and co-workers ${ }^{7}$ in developing a process that has the potential to advance the present state-of-the-art in probe-based nonvolatile memory technology.

Thomas P. Russell and Dong Hyun Lee are in the Department of Polymer Science and Engineering, University of Massachusetts Amherst, Amherst,

Massachusetts 01003, USA.

e-mail: russell@mail.pse.umass.edu

\section{References}

1. Fredrickson, G. H. \& Bates, F. S. Annu. Rev. Mater. Sci. 26, 501-550 (1996)

2. Russell, T. P., Karis, T. E., Gallot, Y. \& Mayes, A. M. Nature 368, 729-731 (1994).

3. Ruzette, A. V. G. et al. Macromolecules 31, 8509-8516 (1998)

4. Ryu, D. Y., Jeong, U., Kim, J. K. \& Russell, T. P. Nature Mater. 1, 114-117 (2002)

5. Ruzette, A. V. G., Mayes, A. M., Pollard, M., Russell, T. P. \& Hammouda, B. Macromolecules 36, 3351-3356 (2003).

6. Ryu, D. Y. et al. Phys. Rev. Lett. 90, 235501 (2003).

. Jo, A., Joo, W., Jin, W.-H., Nam, H. \& Kim, J. K. Nature Nanotech. 4, 727-731 (2009)

8. Ruzette, A. V. G., Banerjee, P., Mayes, A. M. \& Russell, T. P. J. Chem. Phys. 114, 8205-8209 (2001).

9. Coulon, G., Russell, T. P., Deline, V. R. \& Green, P. F. Macromolecules 22, 2581-2589 (1989).

10. Altebaeumer, T., Gotsmann, B., Pozidis, H., Knoll, A. \& Duerig, U. Nano Lett. 8, 4398-4403 (2008).

11. Ryu, D. Y. et al. Macromolecules 37, 3717-3724 (2004).

\title{
OPTOMECHANICAL CRYSTALS
}

\section{Periodic nanobeams bring light and sound together}

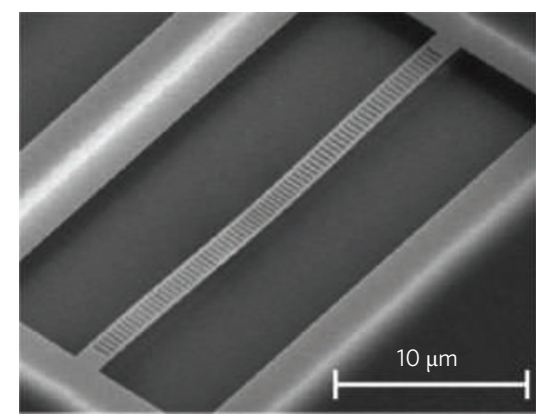

Photonic crystals are periodic structures that guide and trap light, whereas phononic crystals do the same for mechanical vibrations or sound. Oskar Painter and colleagues at the California Institute of Technology have now created optomechanical crystals that can act as both photonic and phononic crystals (Nature 462,
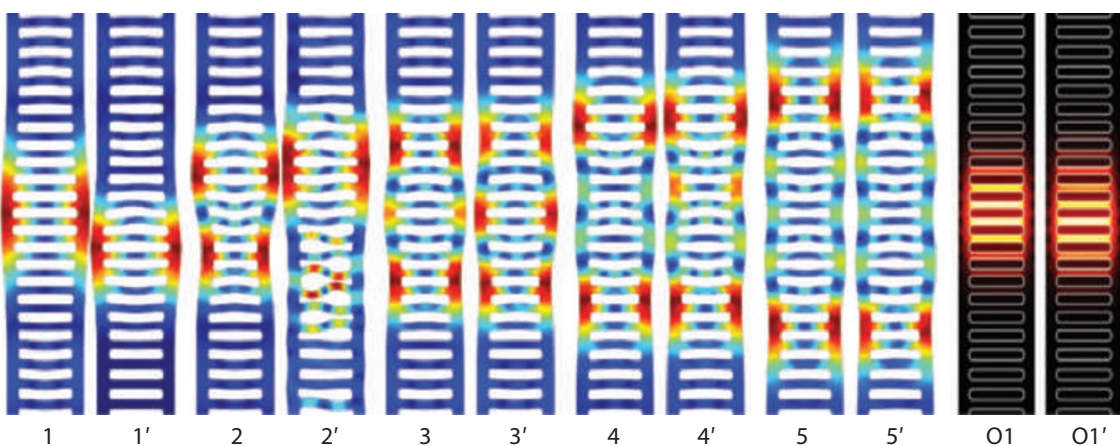

01

$\mathrm{O} 1$

78-82; 2009). These crystals allow light and sound to be confined within a small region.

Painter and co-workers fabricated a ladderlike silicon nanobeam (left) that included a 'defect' in the form of a region at the centre of the beam where the spacing between the rungs in the ladder was reduced. The falsecolour images (right) are simulations showing the first five mechanical modes of the system plus the fundamental optical mode; for each mode the results for the ideal rung spacing in the defect region are shown on the left and the results for the actual spacing are on the right.

OWAIN VAUGHAN 